$$
\begin{array}{rlrl}
\text { Quinhydrone } & \text { Phenoquinone } \\
a_{0}=7.55 \mathrm{~A} . & A_{0}=11.62 \mathrm{~A} . \\
b_{0}=6.05 \mathrm{~A} . & b_{0}=5.97 \mathrm{~A} . \\
\ddot{\beta}_{0}=11.55 \mathrm{~A} . & c_{0}=11.48 \mathrm{~A} . \\
\beta^{\prime}=110^{\circ} & & \beta^{\prime}=109^{\circ}
\end{array}
$$

It may be seen that there is a close similarity between the two sets of cell dimensions, except that the $a$-dimension of quinhydrone is approximately equal to two-thirds of the $A$-dimension of phenoquinone. This factor of two-thirds relating the two dimensions is to be expected in view of the change in stoichiometric composition from 1 quinone/2 phenol to 1 quinone/1 quinol. When this is taken into consideration, it follows that the arrangement and orientation of the molecules must be very similar in the two structures. It therefore appears that the essential structural feature in this type of molecular compound is the plane-to-plane stacking of alternate polarizing and polarizable molecules. Where hydrogen-bonding is possible, as in these two complexes, it must also be of importance in determining the structural arrangement, since it is to realize the full hydrogen-bonding potentialities of the molecules that the change in stoichiometric composition occurs.

In order to investigate the extent to which the principle of plane-to-plane stacking of alternate component molecules determines the structure of molecular compounds, an X-ray crystallographic survey is being made of such series of complexes as those formed by quinones and by aromatic nitro compounds. For all those so far investigated (complexes between hexamethyl-benzene and chloranil, trinitrobenzene and anthracene, trinitrobenzene and naphthalene), it has been possible to propose trial structures based on this principle. The only detailed structure determination which has so far been carried out for a molecular compound formed by trinitrobenzene, namely, with $p$-iodoaniline ${ }^{2}$, also shows this same type of molecular arrangement.

S. C. WALLWORK

T. T. HARDing

University,

Nottingham.

June 27.

1 Anderson, J. S., Nature, 140, 583 (1937).

${ }^{2}$ Fowell, H. M., Huse, G., and Cooke, P. W., J. Chem. Soc., 153 (1943).

\section{Simultaneous Sulphonation and Nitration in an Aromatic Nucleus}

From the literature ${ }^{1,2}$, when $\beta$-naphthylamine is nitrated by either: (1) the addition of potassium nitrate to a cooled solution of $\beta$-naphthylamine in concentrated sulphuric acid; or (2) the addition of ß-naphthylamine nitrate to concentrated sulphuric acid, the products are 5-nitro and 8-nitro 2-naphthylamine.

If, however, $\beta$-naphthylamine is nitrated by the addition of a mixture of nitric and sulphuric acids to a solution of the amine in sulphuric acid at $-25^{\circ}$, or by the addition of $\beta$-naphthylamine sulphate to $a$ mixture of nitric and sulphuric acids, the product is a mixture of two isomeric nitro aminonaphthalene sulphonic acids in the approximate ratio of $70: 30$, the main product being 1-nitro 2-aminonaphthalene 6-sulphonic acid. (The structure of the other isomer is not yet known.)

This reaction is peculiar in four respects : $(a)$ it is the first case known to us of simultaneous sulphonation and nitration in an aromatic nucleus; $(b)$ the main product is the result of ortho-para direction; (c) a remarkable difference in reaction mechanism is produced by comparatively small changes in experimental technique; (d) this anomalous behaviour is not shown by $\alpha$-naphthylamine.

Details of this work will be published elsewhere. A. Bryson

The New South Wales

J. L. Garnetst

University of Technology,

Broadway, Sydney. Sept. 11.

1 Hodgson, H. H., and Davey, W., J. Chem. Soc., 348 (1939).

2 Friedlander and Szymanski, Ber. 25, 2076 (1892).

\section{Degradation of Sugars on lon-Exchange Columns of 'Amberlite IRA $400\left(\mathrm{OH}^{\prime}\right)$ '}

IT has recently been suggested by Roseman et al. ${ }^{\text {" }}$ that columns of 'Amberlite IRA $400\left(\mathrm{OH}^{\prime}\right)$ ' might be used for the separation of certain reducing and non-reducing carbohydrates. Our own experience with this resin suggests that very carefully controlled conditions would be necessary if reliable quantitative results are to be obtained. During an investigation of the non-volatile organic acid components of apple juice and eider, it has been found that sugars are extensively degraded by 'Amberlite IRA 400' used in the $\mathrm{OH}^{\prime}$ form, organic acids being the main products of degradation.

Paper chromatography was used for the separation of the acids following the technique of Lugg and $\mathrm{Overell}^{2}$. Interfering cations, sugars and other nonacidic materials were removed by the use of ionexchange resins, 50-ml. samples of juice or cider being given the following treatments: (i) cation removal on 'Amberlite IR 120', (ii) anion removal on 'Amberlite IRA 400' and elution with $N$ ammonium carbonate, (iii) removal of excess ammonium carbonate by evaporation on the water-bath, and (iv) removal of remaining ammonium ions by treatment with 'Amberlite IR 120'. 10-gm. quantitios of resin retained in glass tubes of $0.8 \mathrm{~cm}$. diameter were used throughout with a flow-rate of $1 \mathrm{ml} . / \mathrm{min}$.

Initially, the 'IRA 400 ' resin was used in the $\mathrm{OH}^{\prime}$ form, and it was found that a number of acid spots were apparent on the chromatograms of treated samples which were not found on those of samples before treatment or after stage (i) in the above procedure (compare chromatograms 2 and 3). Malic, quinic $^{3}$, chlorogenic ${ }^{4}$ and phosphoric acids were identified as present in the juices; ciders contained succinic acid in addition. After ion-exchange treatment, chromatograms revealed five additional spots, two being identified as due to lactic and glycollic acids. Titration of samples after stages (i) and (iv) showed that the anion-exchange treatment resulted in increases of acidity, the extent varying according to the time of contact with the resin. Increases, expressed as malic acid, ranged from 0.05 per cent (in $2 \mathrm{hr}$.) to 0.40 per cent (in $17 \mathrm{hr}$.). Solutions containing a mixture of pure acids, when subjected to the above treatments, showed no change in acidity and the chromatograms showed no additional spots.

An average cider-apple juice contains sugars in the proportions fructose 10 , sucrose 2 and glucose $1 \cdot 5$ per cent ${ }^{5}$. A neutral solution of sugars of this composition was passed through a column of 'IRA $400\left(\mathrm{OH}^{\prime}\right)$ ', the column washed thoroughly with distilled water and 